\title{
Management of Acute Myocardial Infarction and its Effect on Women's Health (Female Versus Male)
}

\author{
Elnaz Asghari 1 , Mohammad Reza Taban Sadeghi 1, Razieh Parizad 1*, \\ Nasrin Mohammasdi jhale ${ }^{1}$
}

\section{Article History:}

Received 13 October 2013

Accepted 3 January 2014

Revised 16 January 2014

Available online 4 February 2014

\section{Keywords: \\ AMI Management \\ Coronary ansiography \\ Gender}

\section{Corresponding Author:}

Razieh Parizad, Cardiovascular

Research Center , Tabriz

University of Medical Sciences,

Tabriz, Iran

Tel: +989143134453

Email: r_parizad2003@yahoo.com

\begin{abstract}
Objectives: Acute Myocardial Infarction (AMI) has traditionally been considered a disease affecting mostly men, yet women are increasingly at risk due to the obesity and diabetes. This study used archival data on hospital discharges from the cardiac care unit (CCU) to examine the AMI risk factors and its management among female patients' presentations at emergency rooms (ER) ended at CCU with a concurrent diagnosis of AMI and to compare the results with men of the same diagnosis.

Materials and Methods: The study was conducted as a prospective cohort survey among patients admitted to CCU. All patients in that ward admitted with documented diagnosis of AMI and positive troponin results were obtained from the study. Some characteristics of the patients such as age, gender, education, risk factors, outcome, cardiac enzymes recorded by one of the researchers at the first day of the admission. Then those patients were followed up until discharge, either referring to another facility or death. Statistical analyses were performed with SPSS for Windows version 13.0 . The data was analyzed by descriptive and inferential statistics.

Results: A total of 182 patients 140 men (68.6\%) and 42 women (20.6\%)) admitted with AMI were enrolled in the study. Women on average had 2 years age difference and had a greater prevalence of hypertension. Men had more previous myocardial revascularization procedures and more often were smokers. There were no difference in previous admission, death, or need for CAG (Coronary angiography).

Conclusion: The awareness needs to be directed towards eliminating the disparities within the female population and heart disease. In this study any important and significant differences were not shown between male and female AMI management.
\end{abstract}

1- Cardiovascular Research Center ,Tabriz University of Medical Sciences, Tabriz, Iran. 


\section{Introduction:}

Coronary arterial disease is the leading cause of mortality and morbidity in developing countries (1). Acute Myocardial Infarction (AMI) has traditionally been considered a disease affecting mostly men, yet women are increasingly at risk due to obesity and diabetes (2).

A recent analysis of the gender-specific prevalence of myocardial infarction (AMI) among subjects aged 35 to 54 years in the United States revealed that during the past 2 decades, the AMI prevalence rates increased among women and decreased among men (3). Given the sparse data on the genderspecific AMI incidence among midlife subjects, it is unclear whether the increase in AMI prevalence among midlife women resulted from an increase in the incidence or from an improvement in survival. (4)

Cardiovascular disease is now recognized as the leading cause of death for women worldwide (1), indeed, more women than men have died annually from ischemic heart disease over the past 2 decades (5). In order to reduce the mortality and morbidity rate associated coronary artery disease within the population and predominantly for females, the focal point should converge on how to improve the knowledge and interpretation of the signs and symptoms of females presenting the AMI, as well as those with increase risk factors of AMI.

It seems that there are increase disparities among females at risk for AMI due to comorbidities and secondary risk factors which proceed to be undiagnosed and treated based on the obscure cardiac signs and symptoms of females(2).

However, some studies have continued to show that gender-related disparities have persisted in the treatment and outcomes of patients with AMI (6). In contrast, others have reported that these perceived differences become insignificant once comorbidities and other confounding factors are accounted for (7). The question of whether gender related disparities truly persist in the management of AMI, as well as the reasons for this presumptive gender gap, is still under debate.(1)

However, current trends in the AMI risk and its management for women are not well understood. This study used archival data on hospital discharges from of the cardiac care unit (CCU) to examine the AMI risk and its management in presentations of female patients from emergency rooms (ER) to CCU and to compare the results with men.

\section{Material \& Methods:}

Since the purpose of this study is to compare AMI outcomes between men and women, the study was conducted as a cohort survey among patients admitted to CCU.

The study was conducted in 2013 and was performed at a National University hospital of heart disease (Shahid Madani) in TabrizIran. The statistical society of this research comprised all AMI patients admitted to CCU. This specialty hospital has three CCU (from 1 to 3 ) and according to its policy, critical AMI patients, after ER, were sent to one of the CCU respectively, so it seems that there could not be any significant differences between three CCUs patients gender and condition. One of the CCUs was selected accidentally, and then all patients admitted in that ward from February to August with documented diagnosis of AMI and positive troponin result were entered the study. After explaining the purpose of the study, documenting anonymous and getting verbal consent, the needed information was gathered by asking the patients and recording their documents by one researcher.

Based on the study design, the census method was used for both male $(n=140)$ and female $(n=42)$ patients. We calculated sample size by using following formula according to a similar study.

$n=\left[\frac{z_{z / 2} \sigma}{\mathrm{E}}\right]^{2}$

\section{Measurements}

Descriptive characteristics of the patients included age, gender, education, major risk factors, cardiac enzymes, drugs, diagnosis and outcome, treatments were recorded by one of the researchers at the first day of the admission, and then those patients were followed up until discharge, either referring to another facility or death. All important interventions like PCI (Percutaneous Coronary Intervention), CABG ( Coronary 
Artery Bypass Graft) and TT ( Thrombolytic Therapy), were recorded too.

\section{Ethical limitations:}

We obtained permissions for the study by getting permission from hospital management and verbal consent of the patients.

\section{Data analysis :}

All statistical analyses were performed with SPSS for Windows version 13.0 .The appropriateness of the scale points to the normal distribution was investigated by a Single Sampling Kolmogorov-Smirnov test. Mean and SD for continuous data, frequencies and percentages for nominal data were used for descriptive statistics analysis. The data was analyzed by descriptive and inferential statistics.

First descriptive statistics of demographic data for the both groups of participants were documented. Then, the results concerning differences between male and female patients from various variables in terms of age and the other results were reported.

When restricting to primary continuous variables, the data were presented as mean \pm standard deviation and categorical variable were expressed as percentages. Comparisons among the baseline and procedural characteristics in men and women were performed using Pearson's Chi-Square test for categorical variables and independent-samples T-test for continuous variables.

Final results were presented as hazard ratios with $95 \%$ confidence intervals. All statistical tests were two-tailed, and a p-value $<0.05$ was considered statistically significant.

\section{Results:}

A total of 182 patients admitted with AMI were enrolled in the study. There were 140 men (68.6\%) and 42 women (20.6\%).Characteristics of the study population stratified by gender are presented in Table 1 and 2. Women were on average 2 years older (64.74 versus 62.21, $\mathrm{P}<0.0001)$, and had a greater prevalence of hypertension (76.2\% vs $44.3 \%, \mathrm{P}<0.0001)$. Men more often were smokers $(45.7 \%$ vs $11.9 \%, \mathrm{P}<0.0001)$. There was no difference in previous admission, death, or need for revascularization. Length of stay was longer in women $(1.72 \pm 2.78$ days vs $4.37 \pm 3.14$ days, $p<0.013)$. The same rate of adherence to dual antiplatelet therapy was observed Similarly rate of stent thrombosis was similar according to gender.

\section{Discussion:}

This study evaluated similarities and differences between men and women management, who hospitalized with AMI and determined if any differences can be explained on the basis of gender.

In the present study on the management of women and men, the main findings were as follows:

1. Women and men had not similar risk factors.

2. Women reached medical help sooner.

3. Gender was not related to type of drug used in hospital.

4. Use of CABG or PCI procedures in this clinical setting was not associated with the patients' gender.

Heart attacks were considered to predominately affect the male population (8). This is shown in the study too, although the rate of female patients was higher in compare with some studies (7), most patients admitted to CCU were male (68.6\%). This is similar to Gulf RACE and GRACE studies $(9,10)$. As technology has improved, and tests are more accurate and available, health care professionals have more tools at their disposal, and are able to provide better diagnosis and administer more appropriate and timely treatment compared to past decades. This is perhaps one reason why there's been an increase in the number of females diagnosed with heart disease; so past disparities in recognizing AMI in women according to signs and symptoms decreases; in the other words, in the past, diagnosis was based on signs and symptoms, and because of different signs and symptoms of MI between male and female, they couldn't diagnose MI in women as soon, but nowadays that disparities could not be seen because of better diagnostic tools.

In this study Women presenting with AMI were older than men, this is similar to Gulf Race, Sulaiman et al, Grace and FathOrdoubadi et al studies $(11,12,13$, and 
14).That could be the result of protecting effect of premenopausal female hormones. While there are some controllable risk factors such as dyslipidemia, diabetes, hypertension and obesity, cardiac disease is still the number one killer in women (15). In the worldwide inter-heart study of patients from 52 countries, nine potentially modifiable risk factors accounted for over $94 \%$ of the population-attributable risk of a first Acute Myocardial Infarction (AMI) in women. These factors included smoking, dyslipidemia, hypertension, diabetes, abdominal obesity, psychosocial factors (e.g., depression, perceived stress, and life events), daily consumption of fruits and vegetables, regular alcohol consumption, and regular physical activity (16). In this study hypertension in women and smoking in men were more. But other risk factors were similar between them. In FathOrdoubadi et al study, the difference between men and women risk factors were significant in smoking and hypertension; smoking was more in men but another was high in female(14). The explaining these differences need to assess cultural varieties and therefore related factors like nutritional and activity habits.

In some studies Women and the elderly take longer to seek medical care when acute coronary syndrome (AMI) symptoms start (12). One possible reason for women's delayed arrival to the emergency department and for less aggressive triage and care in the emergency department is that MI symptoms differ between men and women. Some studies showed women patients with MI are less likely to present with ischemic chest pain $(9,10)$ According to previous studies the delayed diagnosis and treatment of AMI in females may have increased the morbidity and mortality rate in females (4).In this research, women reached ED significantly sooner. Some research revealed that when patients finally present at the emergency department for management of AMI, women have further delays in the completion of an electrocardiogram and in the initiation of life-saving therapy, i.e., thrombolytic or balloon angioplasty(2). Controversially, here, there were not seen any differences in this term. It could be explained that the signs and symptoms of both genders were assessed.

In this study, the rate of death and final interventions was similar between two groups. Our findings are in contrast to several previous studies in which women compared to men had markedly lower rates of procedural success and worse in-hospital outcomes including mortality (17). That may be the result of the accuracy of laboratory results or the continuously presence of experienced cardiologists in ED. The on time arrival of the female patients could be another reason for this finding, it means the sooner the presence in ED, the more time for assessing and treatment.

\section{Conclusion :}

The extent of time from the onset of the symptoms to the time of treatment is critical for enhanced positive outcomes. The awareness needs to be directed towards eliminating the disparities within the female population and heart disease. In acknowledging and recognizing the differences of AMI management between the genders the healthcare professionals will be further competent towards accurately diagnosing and treating males and females in a consistent approach as a result of correctly diagnosing and providing timely intervention.

The quality of care problems related to lack of recognition of the increasing AMI risk for women may be exacerbated by the fact that AMI may show substantially different symptoms in women that those which are commonly accepted for men. Thus it is important to understand and document any increasing AMI risk for women who present to emergency rooms. A delay in a diagnosis of AMI increases a woman's risk of death, as well as the potential for her having substantial sequels from the AMI.

This study improves our understanding of the magnitude of AMI management for women. While epidemiological reports on rates of AMIs for men and women are routinely published by the Centers for Disease Control (CDC), these do not examine the quality of AMI management, so the importance of this study gets obvious. According to the results the quality of AMI 
management in the main heart center of the province for both men and women is similar and any disparities, against most other researches, were seen.

\section{Conflicts of interest:}

The authors declare no conflict of interest in this study .

\section{Acknowledgments:}

The authors would like to thank all of the patients who participated indirectly in this study and also thank Tabriz medical science university and hospital managers who supported the research.

Table 1. Baseline clinical characteristics of women and men presenting with MI.

\begin{tabular}{lllll}
\hline & Characteristics & Men $(\mathbf{n}=140)$ & Women $(\mathrm{n}=42)$ & P-value \\
\hline Education & Illiterate & $99(71.7)$ & $33(78.6)$ & NS \\
& Not University & $28(20.3)$ & $7(16.7)$ & NS \\
& Diploma & $1(0.7)$ & $1(2.4)$ & NS \\
& University & $1(0.7)$ & $0(0)$ & NS \\
& MS Or Phd Degree & $5(3.6)$ & $1(2.4)$ & $<0.0001$ \\
& Hypertension & $62(44.3)$ & $32(76.2)$ & NS \\
& Diabetes Mellitus & $41(29.3)$ & $11(26.2)$ & $<0.0001$ \\
& Hyperlipidemia & $23(16.4)$ & $10(23.8)$ & NS \\
& Current Smoker & $64(45.7)$ & $5(11.9)$ & NS \\
& Past CABG & $6(4.3)$ & $2(4.8)$ & NS \\
& Past PCI & $13(9.3)$ & $3(7.1)$ & NS \\
& Age( mean \pm SD), & $62.20 \pm 12.55$ & $64.73 \pm 11.81$ & \\
& (years) & & & $<0.013$ \\
& From Home To ED & $6.36 \pm 10.36$ & $4.10 \pm 4.24$ & \\
(Hours) & & & NS \\
& From ED To CCU & $2.18 \pm 1.72$ & $2.21 \pm 1.98$ & NS \\
\hline
\end{tabular}

NS =non-significant; SD =standard deviation; $\mathrm{MI}=$ myocardial infarction; $\mathrm{PCI}=$ percutaneous coronary intervention; $\mathrm{CABG}=$ coronary artery bypass surgery; and Percents are column percentages. All values are n (\%) unless specified otherwise.

It seems that women reached to ED sooner and Hypertension and smoking are the main differences. 
Figure 1. MI according to ST elevation present.

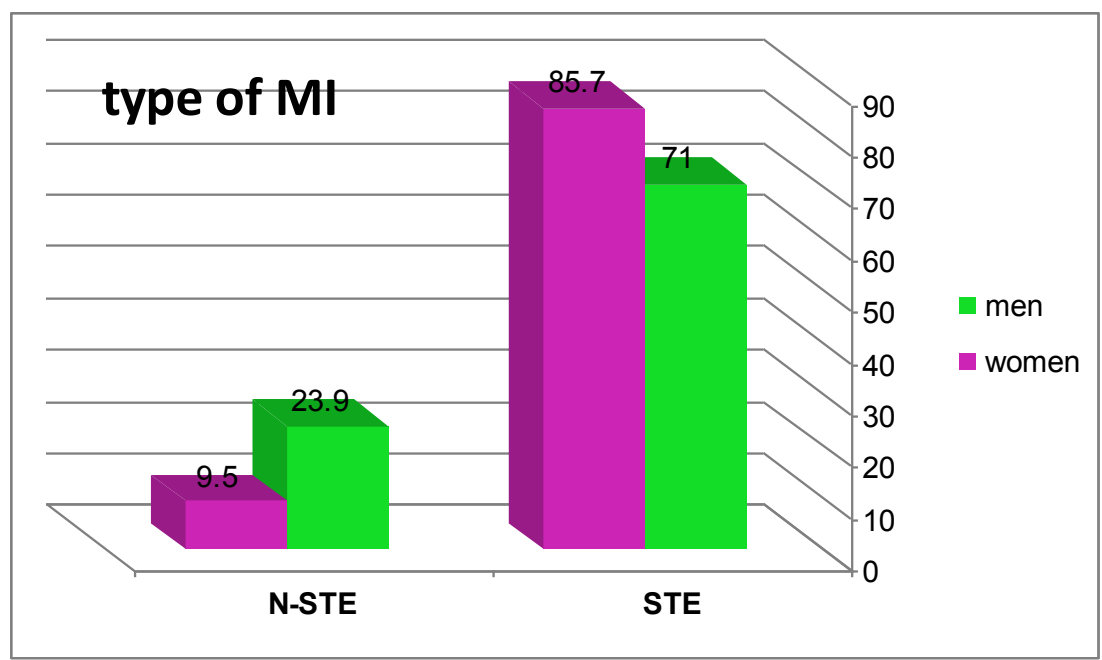

STE: MI with ST segment elevation. N- STE: MI without ST segment elevation. Data are presented in percent.

ST elevation MI seems prevalent in both genders and both genders are similar in type of MI.

Figure 2. Type of ST elevation MI according to the location.

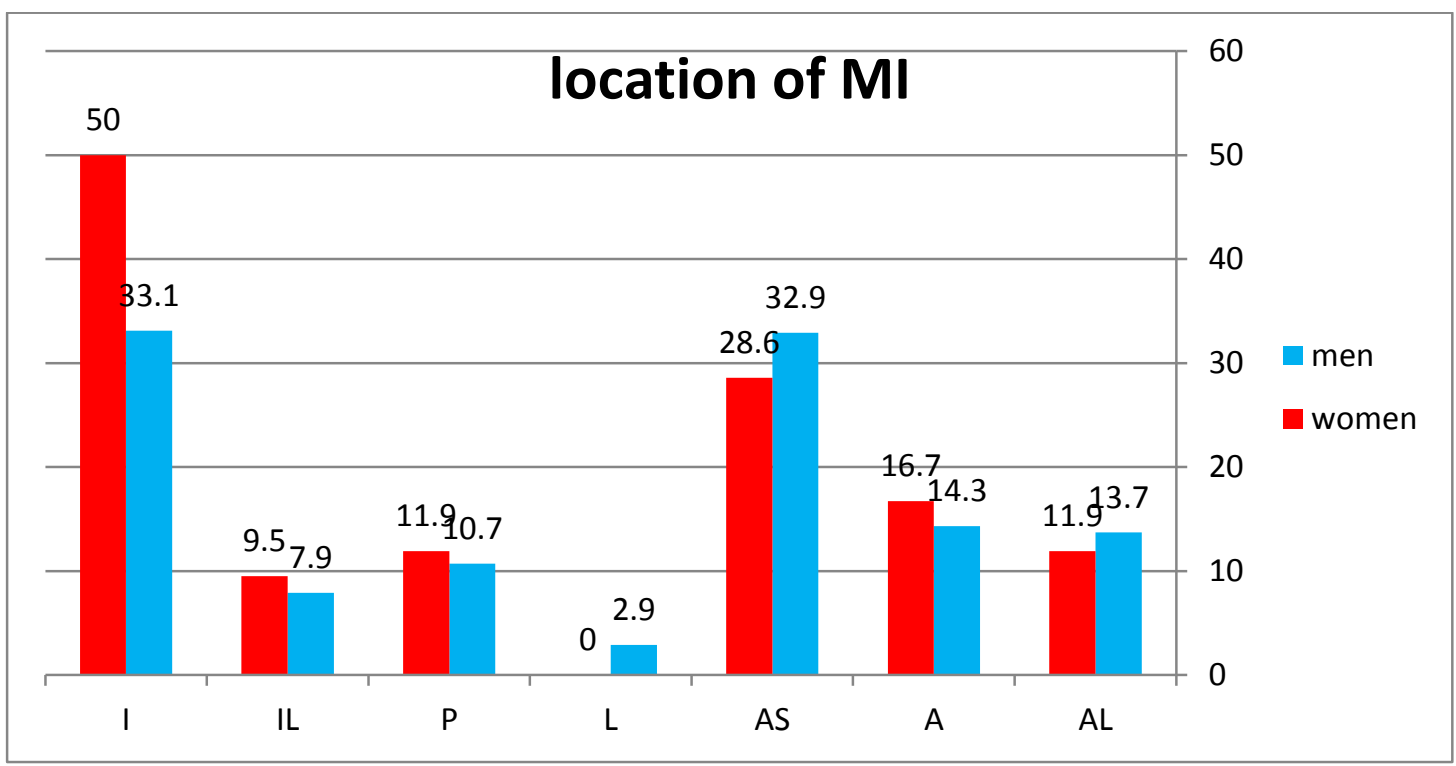

I: inferior MI, IL: inferio-lateral MI, P: posterior MI, L: lateral MI, AS: anterio- septal MI, A: anterior MI, AL: anterio-lateral MI. All is in percentage. 
Apparantly Inferior MI seems more prevalent in all samples but any statistical differences are shown between groups.

Figure 3. Usage of drugs during treatment

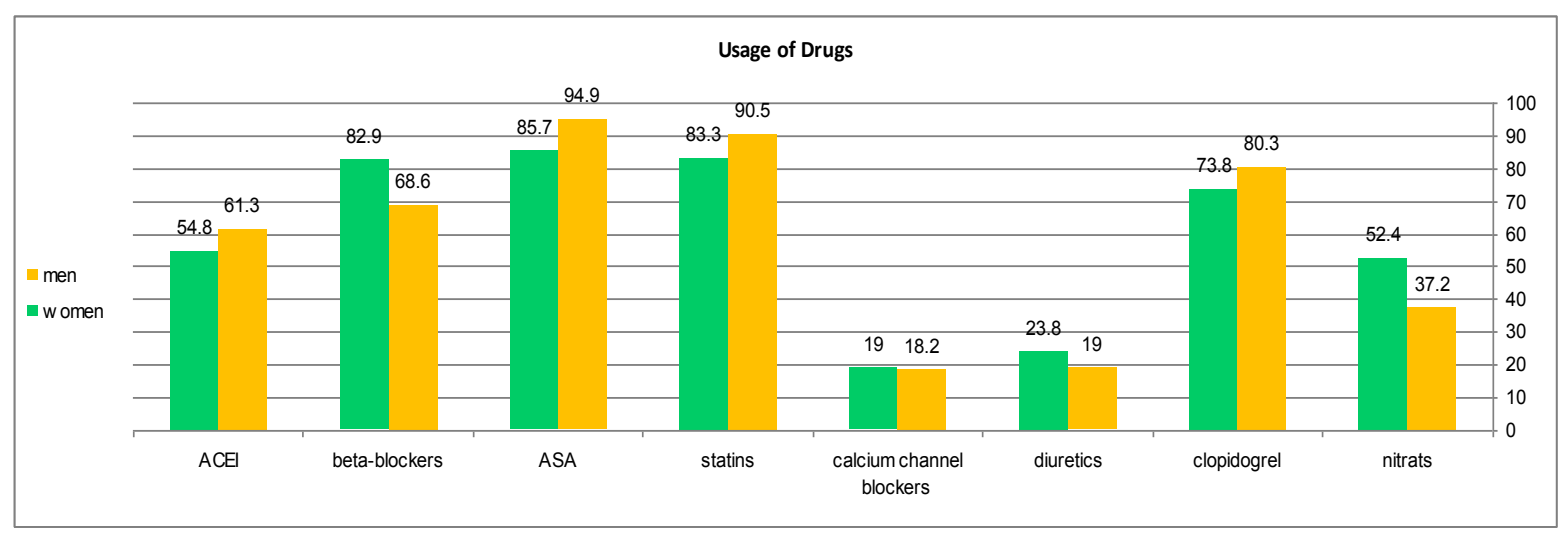

There are any statistical differences in used drugs between male and female.

Table 2. Final results of hospitalization

\begin{tabular}{|c|c|c|c|c|c|c|}
\cline { 3 - 6 } \multicolumn{2}{c|}{} & \multicolumn{2}{c|}{ Male } & \multicolumn{2}{c|}{ Female } & \multirow{2}{*}{ P value } \\
\cline { 3 - 6 } \multicolumn{2}{c|}{} & Frequency & Percent & Frequency & Percent & \\
\hline \multirow{3}{*}{$\begin{array}{l}\text { Invasive } \\
\text { Treatment }\end{array}$} & TT & 49 & 35.8 & 10 & 23.8 & NS \\
\cline { 2 - 6 } & PCI & 48 & 34.3 & 18 & 42.9 & NS \\
\cline { 2 - 6 } & CABG & 6 & 4.3 & 1 & 2.4 & NS \\
\hline \multicolumn{2}{|r|}{ Discharge } & 127 & 90.7 & 39 & 92.9 & NS \\
\hline \multicolumn{2}{|c|}{ Expire } & 13 & 9.3 & 3 & 7.1 & NS \\
\hline
\end{tabular}

$\mathrm{NS}=$ non-significant; $\mathrm{PCI}=$ percutaneous coronary intervention; $\mathrm{CABG}=$ coronary artery bypass surgery; TT $=$ Thrombolytic therapy

Assessed final results of hospitalization for both groups were statistically similar. 


\section{References:}

1. Poon S, Bierman AS, Grondin FR, Goodman SG, Eagle KA, Yan RT, et al. Bridging the gender gap: Insights from a contemporary Analysis of sex-related differences in the treatment and Outcomes of patients with acute coronary syndromes. American Heart Journal. 2012; 163(1):66-73.

2. Arslanian-Engoren C, Patel A, Fang J, Armstrong D, Kline-Rogers E, Duvernoy CS, et al. Symptoms of Men and Women Presenting With Acute Coronary Syndromes. The American Journal of Cardiology. 2006; 98:1177-81.

3. Towfighi A, Zheng L, Ovbiagele B. Sex-specific trends in midlife coronary heart disease risk and prevalence. Arch Intern Med. 2009; 169:1762-6.

4. Markovic D, Ovbiagele B, Duvernoy CS. National Gender-Specific Trends in Myocardial Infarction Hospitalization Rates Among Patients Aged 35 to 64 Years. The American Journal of Cardiology. 2011; 108:1102-7.

5. American Heart Association. Heart disease and stroke statistics: 2010. update. Available at: http://www.americanheart.org/downloadable/heart/1265665152970DS3241\%

6. Bugiardini R, Yan A, Yan R. Factors influencing underutilization of evidence based therapies in women. Eur Heart J. 2011; 32:1337-44.

7. Berger J, Elliott L, Gallup D. Sex differences in mortality following acute coronary syndromes. 2009:20874-82.

8. Bertoia ML, Allison MA, Manson JE, Freiberg MS, Kuller LH, Solomon AJ, et al. Risk Factors for Sudden Cardiac Death in Post-Menopausal Women. Journal of the American College of Cardiology. 2012:2674-82.

9. JSuwaidi MA. Impact of gender in patients with acute coronary syndrome. Expert Rev Cardiovasc. 2009; 7(4):411-21.v

10. Race G, Gaziano TA, Bitton A, Anand S, Abrahams-Gessel S, Murphy A. Growing epidemic of coronary heart disease in low-and middle-income countries. Curr Probl Cardiol $2010 ; 35: 72-115$.

11. Dey S, Flather, Devlin G, Brieger, D. G, E.P. S, P.G. F, G. J, et al. For the GRACE Investigators. Sex-related differences in the presentation, treatment and outcomes among patients with acute coronary syndromes: the Global Registry of Acute Coronary Events. Heart. 2009; 95(1):20-6.

12. Sulaiman K, Panduranga P, Al-Zakwani I. Gender-related diferences in the presentation,Management, and outcomes among patients with acute coronary syndrome from Oman. Journal of the Saudi Heart Association. 2011 ; 23:17-22.

13. Grace N,Akhter, N, Milford-Beland, S, Roe, M.T, Piana, R.N, Kao, J,Shroff, A. Gender differences among patients with acute coronary syndromes undergoing percutaneous coronary 
interven-tion in the American College of Cardiology-National Cardiovas-cular Data Registry (ACC-NCDR). Am. Heart J. 2009; 157 (1), 141-148

14. Fath Ordoubadi M, Hadaegh F, Harati H, and Ghanbarian A, Azizi F Sadeghi M, Ruhafza H, Shirani Sh, Akhavan. Prevalence of coronary heart disease among Tehran adults: Tehran lipid and glucose study. East Mediterr Health J 2009; 15:157-166. [6]

15. Wild R, Carmina E, Diamanti-Kandarakis E, Dokras A, Escobar-Morreale H, Futterweit $\mathrm{W}$, et al. Assessment of cardiovascular risk and prevention of cardiovascular disease in women with the polycystic ovary syndrome: a consensus statement by the Androgen Excess and Polycystic Ovary Syndrome (AE-PCOS) Society. J Clin Endocrinol Metab. 2010; 95(5):203849.

16. Dokras A. Cardiovascular disease risk in women with PCOS. Steroids. 2013;78: 773-6.

17. Onuma Y, Kukreja N, Daemen J, Garcia-Garcia H, Gonzalo N, Cheng J, et al. Interventional Cardiologists of Thoraxcenter. Impact of sex on 3-year outcome after percutaneous coronary intervention using bare-metal and drugeluting stents in previously untreated coronary artery disease: insights From the RESEARCH (Rapamycin-Eluting Stent Evaluated at Rotterdam Cardiology Hospital) and T-SEARCH (Taxus-Stent Evaluated at Rotterdam Cardiology Hospital) registries. JACC Cardiovasc Interv. 2009;2:603- 10. 